\title{
BRIGHT LOCALIZED NEAR-INFRARED EMISSION AT 1-4 AU IN THE AB AURIGAE DISK REVEALED BY IOTA CLOSURE PHASES
}

\author{
R. Millan-Gabet, ${ }^{1}$ J. D. Monnier,${ }^{2}$ J.-P. Berger, ${ }^{3}$ W. A. Traub,${ }^{4,5}$ F. P. Schloerb,${ }^{6}$ E. Pedretti,${ }^{2}$ M. Benisty, ${ }^{3}$ \\ N. P. Carleton, ${ }^{4}$ P. Haguenauer,${ }^{5}$ P. Kern, ${ }^{3}$ P. Labeye, ${ }^{7}$ M. G. Lacasse, ${ }^{4}$ F. Malbet ${ }^{3}$ \\ K. Perraut, ${ }^{3}$ M. Pearlman, ${ }^{4}$ and N. Thureau ${ }^{2}$ \\ Received 2006 May 1; accepted 2006 May 23; published 2006 June 28
}

\begin{abstract}
We report on the detection of localized off-center emission at 1-4 AU in the circumstellar environment of the young stellar object $\mathrm{AB}$ Aurigae. We used closure-phase measurements in the near-infrared that were made at the long-baseline interferometer IOTA, the first obtained on a young stellar object using this technique. When probing sub-AU scales, all closure phases are close to zero degrees, as expected given the previously determined size of the $\mathrm{AB}$ Aurigae inner-dust disk. However, a clear closure-phase signal of $-3.5 \pm 0.5$ is detected on one triangle containing relatively short baselines, requiring a high degree of non-point symmetry from emission at larger (AU-sized) scales in the disk. We have not identified any alternative explanation for these closure-phase results, and we demonstrate that a "disk hot spot" model can fit our data. We speculate that such detected asymmetric near-infrared emission might arise as a result of localized viscous heating due to a gravitational instability in the $\mathrm{AB}$ Aurigae disk, or to the presence of a close stellar companion or accreting substellar object.
\end{abstract}

Subject headings: infrared: stars — instrumentation: interferometers -

planetary systems: protoplanetary disks — stars: formation -

stars: individual $(\mathrm{AB}$ Aurigae $)$ - techniques: high angular resolution

\section{INTRODUCTION}

AB Aurigae (A0 Ve, $d=144 \mathrm{pc}, V=7.1, H=5.9$ ) is often referred to as the prototype of the Herbig $\mathrm{Ae} / \mathrm{Be}(\mathrm{HAeBe})$ class of pre-main-sequence stars of intermediate mass. Like their solar-type analogs, the T Tauri objects, HAeBe objects are known to be surrounded by preplanetary disks of gas and dust. AB Aur was one of the first young stellar objects (YSOs) to be spatially resolved in the near-infrared (NIR; Millan-Gabet et al. 1999), using the technique of long-baseline optical interferometry. These and subsequent observations of a relatively large sample of HAeBe (and T Tauri) objects found that essentially all objects have characteristic NIR sizes much larger than expected from previous generation disk models that were tuned to fit the spectrophotometric data alone (Akeson et al. 2000; Tuthill et al. 2001; Millan-Gabet et al. 2001; Akeson et al. 2002; Eisner et al. 2004; Akeson et al. 2005; Eisner et al. 2005; Monnier et al. 2005). These results have prompted in part a significant revision of disk models, whereby previously ignored detailed physics of the disk inner edge are now incorporated with some success (Natta et al. 2001; Dullemond et al. 2001; Monnier \& Millan-Gabet 2002; Muzerolle et al. 2003; Isella \& Natta 2005). For a recent review of these developments, the reader is also referred to Millan-Gabet et al. (2006).

A common characteristic of all the interferometer studies mentioned is that they were based on single-baseline data, and there-

\footnotetext{
${ }^{1}$ Michelson Science Center, California Institute of Technology, MS 100-22, Pasadena, CA 91125; rafael@ipac.caltech.edu.

${ }^{2}$ University of Michigan, Astronomy Department, Ann Arbor, MI 48109-1090.

${ }^{3}$ Laboratoire d'Astrophysique de Grenoble, 414 Rue de la Piscine, 38400 Saint Martin d'Heres, France.

${ }^{4}$ Harvard-Smithsonian Center for Astrophysics, Cambridge, MA 02138.

${ }^{5}$ Jet Propulsion Laboratory, California Institute of Technology, Pasadena, CA 91109.

${ }^{6}$ University of Massachusetts at Amherst, Astronomy Department, Amherst, MA 01003.

${ }^{7}$ Laboratoire d'Electronique de Technologie de I'Information (LETI), CEA, 17 rue des Martyrs, 30054 Grenoble, France.
}

fore depended on the interpretation of only visibility amplitudes and with very sparse spatial frequency coverage. One of the unexplored frontiers in optical long-baseline interferometry, particularly in the area of YSO science, is the measurement and interpretation of not only visibility amplitudes but also closure phases (CPs), obtained at arrays containing three or more telescopes. Contrary to the visibility amplitudes or phases measured for baseline pairs, the $\mathrm{CP}$ is uncorrupted by atmospheric turbulence (Jennison 1958; Monnier 2003) and can be calibrated to higher precision. The $\mathrm{CP}$ can have values different from 0 or $180^{\circ}$ only for a non-point-symmetric sky brightness and is therefore a highly effective probe of this type of morphology. Moreover, $\mathrm{CP}$ and visibility amplitude data may be used together to provide powerful constraints to parametric models describing relatively complex morphologies. In principle, given sufficient spatial frequency coverage, direct image reconstruction becomes possible, as is commonly done at radio wavelengths (Readhead et. al. 1980; Cornwell \& Wilkinson 1981).

The new models of HAe objects, and of AB Aur in particular, successfully reproduce the spectral features and broadband spectral energy distribution (SED) with a model in which no or little optical depth exists between the central star and the dust disk inner edge, such that the frontal stellar heating forms a hot wall of emission from which essentially all the NIR (disk) flux arises. This model is also successful at reproducing the characteristic NIR sizes measured interferometrically. Moreover, the outer regions of circumstellar disks are believed to have scale heights that increase with radius (flaring; Kenyon \& Hartmann 1987). Thus, non-point-symmetric brightness distributions and nonzero CPs are expected for star-disk systems such as AB Aur if they have some inclination to the observer's line of sight, as the cooler foreground disk regions occult part of the hotter inner disk regions (see also Tuthill et al. 2001, Malbet et al. 2001, and Isella \& Natta 2005). The original goal of the work presented here was to search for such skewed emission coming from the inner-dust disk; and in a forthcoming publication (Monnier et al. 2006), we discuss in detail the results of our survey of 14 


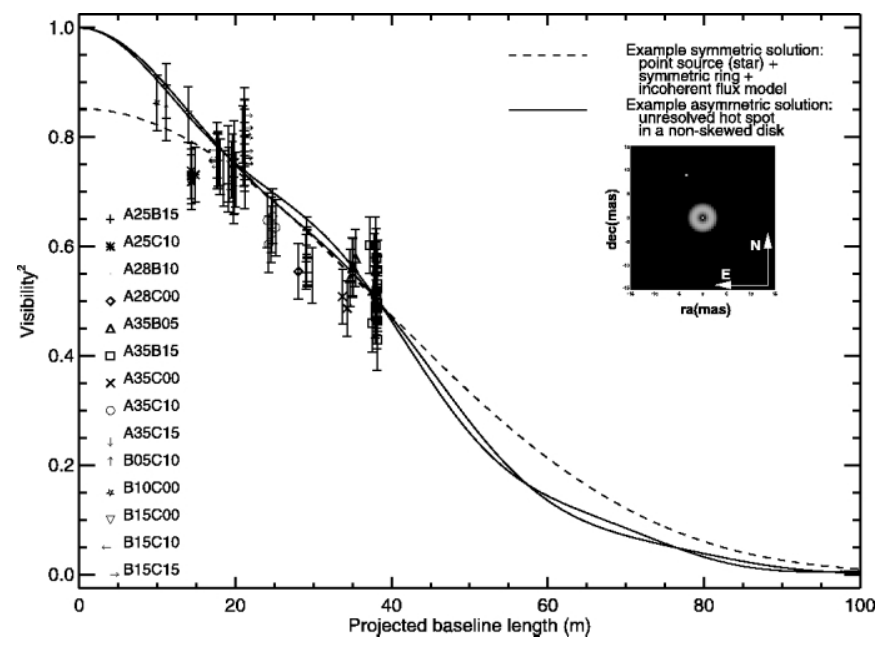

FIg. 1.-Calibrated $V^{2}$ data as a function of projected baseline length, and comparison to the two models discussed in the text. For the asymmetric model, the plot shows cuts in the $(u, v)$-plane along two distinct directions that encompass most of the $\mathrm{AB}$ Aur data. As discussed in the text, a unique solution for the asymmetric model is not well constrained; in order to illustrate the geometry, the inset image $(30 \times 30$ mas $)$ shows the example solution for which the model curves are calculated.

YSOs in the context of hot inner-dust wall models. In this Letter, we report instead on the unexpected detection of a strong CP signal that originates farther out in the AB Aur disk.

\section{OBSERVATIONS AND DATA ANALYSIS}

The data presented here were obtained at the upgraded threetelescope Infrared Optical Telescope Array (IOTA; Traub et al. 2003), using the new integrated-optics (IO) $H$-band combiner IONIC-3 (Integrated Optics Near-infrared Interferometric Camera; Berger et al. 2003). IOTA/IONIC-3 has been in routine operation since 2002 December, and the first scientific results were published in Monnier et al. (2004) and Kraus et al. (2005).

The three IOTA telescopes are moveable along two orthogonal linear arms; telescopes A and C can move along a 35 m northeast arm, while telescope B moves along a $15 \mathrm{~m}$ southeast arm. This allows an aperture of $35 \mathrm{~m} \times 15 \mathrm{~m}$ to be synthesized, giving a maximum physical baseline $B=38 \mathrm{~m}$. For the AB Aur declination $\left(\delta=30^{\circ}\right)$, the projected baselines are in the range $B_{p}=$ $10-38 \mathrm{~m}$, providing spatial resolution $\sim \lambda_{0} /\left(2 B_{p}\right)=4-17$ mas (or 0.6-2.4 AU) in the $H$ band $\left(\lambda_{0}=1.65 \mu \mathrm{m}\right)$. The IONIC-3 optical circuit acts to split the light from each telescope before recombining each telescope pair $(\mathrm{AB}, \mathrm{BC}, \mathrm{AC})$ at three $\mathrm{IO}$ couplers, leading to six interferometric channels (two for each baseline).

The work presented here is based on observations made over the period 2002 December-2004 December, comprising a total of 18 epochs, 14 two-telescope configurations (visibility amplitude data), and six three-telescope configurations (CP data). As is standard practice, observations of $\mathrm{AB}$ Aur were interspersed with observations of nearby calibrator stars to measure slowly varying system visibilities and CPs. We used the calibrator stars HD 32406 (K0 III, $V=6.2, H=3.6$ ) and HD 31233 (K0, $V=7.2, H=4.6$ ), of uniform-disk angular diameters $1.2 \pm 0.7$ and $0.6 \pm 0.4$ mas, respectively. The observations presented here were made using a standard $H$-band filter $\left(\lambda_{0}=1.65 \mu \mathrm{m}, \Delta \lambda=0.30 \mu \mathrm{m}\right)$ and in unpolarized light.

Reduction of the visibility amplitude data was carried out using a custom implementation of the method outlined by Coudé du Foresto et al. (1997) and already described and dem-
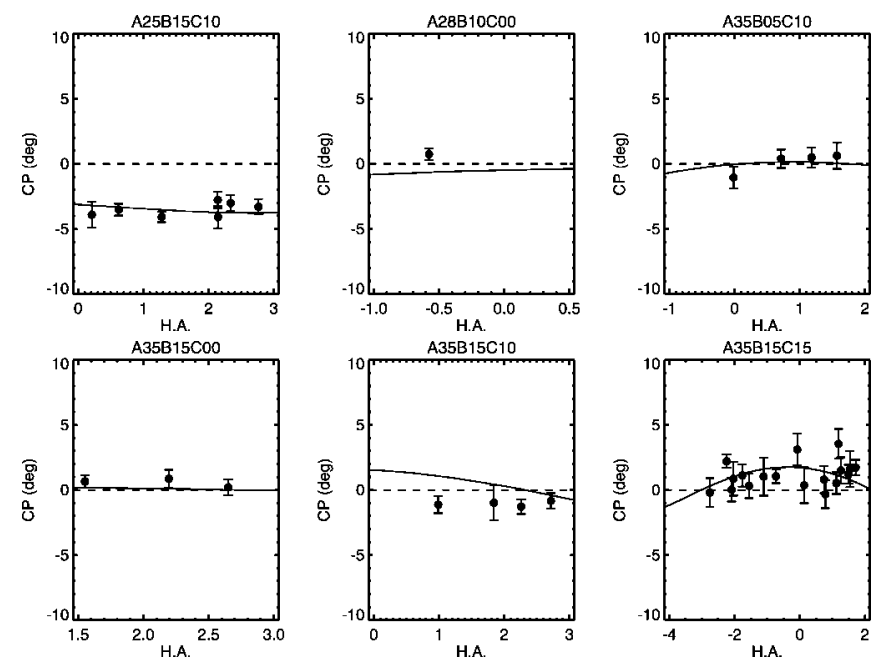

FIG. 2.-Calibrated CP data as a function of hour angle, and comparison to the models discussed in the text.

onstrated in Monnier et al. (2004). To ensure a good CP measurement, we required that interferograms be detected on $\geq 2$ baselines, a condition nearly always maintained by a real-time fringe packet "tracker" (Pedretti et al. 2005). Based on studies of our absolute night-to-night calibration accuracy, we adopt a typical systematic error of $\sigma_{V^{2}}=0.05$ (e.g., $2.5 \%$ error in visibility for unresolved sources); this error has been combined (in quadrature) with the statistical error for plotting and fitting purposes. We followed the method of Baldwin (1996) for calculating the complex triple amplitude in deriving the $\mathrm{CP}$, explicitly guaranteeing "fringe frequency closure" $\left(\nu_{A B}+\nu_{B C}+\right.$ $\nu_{C A}=0$ ). Chromaticity effects are the main limitation to our absolute precision when the calibrator and source are of very different spectral types because of the different effective wavelengths. While we minimize these errors by using calibrators of spectral type relatively similar to that of the target, a conservative systematic error of $\sigma_{\mathrm{CP}}=0.5$ has been adopted here. Further details on our data reduction and calibration methods are given in Monnier et al. (2006). The $V^{2}$ and CP data have been averaged in the two-dimensional spatial frequency $(u, v)$-plane using an averaging interval of $4 \mathrm{~m}$, resulting in 75 and 37 independent $V^{2}$ and $\mathrm{CP}$ measurements, respectively. The calibrated $V^{2}$ and CP data are available from the authors ${ }^{8}$ in the OI-FITS format (Pauls et al. 2005).

The $V^{2}$ and CP data are shown in Figures 1 and 2, along with models discussed in the next section. The main characteristics of our AB Aur data that underlie the principal results presented in this Letter are as follows: (1) A definite CP signal is detected $\left(-3^{\circ}\right.$ to $\left.-4^{\circ}\right)$, which surprisingly appears in a triangle of relatively short baselines (triangle A25B15C10), ${ }^{9}$ indicating some degree of non-point symmetry on relatively large spatial scales (1-4 AU; see $\S 3$ ). (2) The CP data are consistent with zero on all other baseline triangles (Fig. 2), indicating a high degree of point symmetry on sub-AU spatial scales for $A B$ Aur. (3) In spite of including baselines of varying orientations, the curve traced by the $V^{2}$ versus baseline length data (Fig. 1) is relatively smooth, indicating that the NIR brightness is also largely cir-

\footnotetext{
${ }^{8}$ The data can also be found in the data archives section of the Optical Long Baseline Interferometry News (OLBIN) Web site, http://olbin.jpl.nasa.gov/.

${ }^{9}$ The notation AxxByyCzz indicates an IOTA configuration in which telescope $\mathrm{A}$ is located at the station that is $\mathrm{xx}$ meters from the array origin, located at the corner of the L-shaped track, and similarly for telescopes B and C.
} 
TABLE 1

Results from FitTing to Disk Hot Spot Model

\begin{tabular}{|c|c|c|c|c|c|c|}
\hline \multirow[b]{2}{*}{ Model Description } & \multicolumn{3}{|c|}{ Fraction of Light } & \multirow[b]{2}{*}{ Disk Properties } & \multirow[b]{2}{*}{ Spot Properties } & \multirow{2}{*}{$\begin{array}{l}\text { REDUCED } \chi^{2} \\
\left(V^{2}, \mathrm{CP}\right)\end{array}$} \\
\hline & Star & Disk & Spot & & & \\
\hline $\begin{array}{l}\text { Unresolved hot spot } \\
\text { with non-skewed disk }\end{array}$ & 0.3 & 0.68 & 0.02 & $\begin{array}{l}\text { Ring diameter } 3.6 \text { mas, } \\
\text { ring width/diameter } 0.25 \\
\text { Ring diameter } 3.1 \mathrm{mas},\end{array}$ & $\begin{array}{l}\text { Unresolved spot, } \\
r_{\mathrm{G}}=9 \text { mas at P.A. } 22^{\circ}\end{array}$ & 1.5 \\
\hline $\begin{array}{l}\text { Gaussian hot spot } \\
\quad \text { with skewed disk } \ldots \ldots \ldots . .\end{array}$ & 0.3 & 0.62 & 0.08 & $\begin{array}{l}\text { ring width/diameter } 0.5 \text {, } \\
\text { max. skew }=1.0 \text { at P.A. } 172^{\circ}\end{array}$ & $\begin{array}{l}\text { Gaussian FWHM } 12 \text { mas, } \\
r_{\mathrm{G}}=29 \text { mas at P.A. } 12^{\circ}\end{array}$ & 1.8 \\
\hline
\end{tabular}

NotE.-See description of all parameters in $\S 3$. Error bars are not presented in this table since multiple local $\chi^{2}$ minima exist with comparable probabilities. Thus, these results are representative of parameters that can fit the nonzero closure phase and the visibilities, but are not unique. More short-baseline data will be needed to confirm the "disk hot spot" model and to unambiguously determine the hot spot location.

${ }^{a}$ The predictions of this model are compared to the data in Figs. 1 and 2.

cularly symmetric on small scales (consistent with previous interferometer observations). (4) The $V^{2}$ versus baseline length curve traced by the data does not appear to extrapolate to 1.0 at zero spatial frequency, implying the presence of an extended component contributing a small fraction of the $H$-band flux ( $8 \%$ if it were completely resolved by the shortest IOTA baselines, $B_{p}=9 \mathrm{~m}$ ). We note that of the 14 YSOs in our CP survey (Monnier et al. 2006), AB Aur is unique in showing nonzero CP simultaneous with such high visibilities.

\section{MODELING AND RESULTS}

The $(u, v)$-coverage obtained in these observations is relatively dense, unprecedented for YSO observations using an optical interferometer. However, it still contains significant gaps, a pronounced north-south bias, and, even for the longest IOTA baselines, AB Aur appears only partially resolved $\left(V_{\min }^{2} \sim 0.4\right)$, all of which frustrate attempts to directly reconstruct a meaningful image from the $V^{2}$ and $\mathrm{CP}$ data.

We have thus turned to parametric imaging in order to interpret our observations. Motivated by the inner-dust wall models introduced in $\S 1$, we begin by modeling the AB Aur NIR brightness as a central star plus a Gaussian ring, of inner diameter $D_{\text {ring }}$ and width $W_{\text {ring }}$ (expressed as a fraction of the inner ring diameter). We use previous results based on SED decomposition establishing that the central star in $\mathrm{AB}$ Aur contributes $\sim 30 \%$ of the $H$-band flux and is unresolved (pointlike) to the IOTA baselines (Millan-Gabet et al. 2001) — the exact stellar fractional flux is not relevant to the conclusions of this Letter. Although the visibility amplitude data allow us to make a direct measurement of the ring diameter, they do not constrain the ring width well, and we follow previous workers in adopting a 25\% fractional width for the ring (also not critical to our conclusions). Using fits of elliptical rings (representing inclined inner disks) to the $V^{2}$ data, we find near-unity major-axis/minoraxis ratios, i.e., small disk inclinations $\$ 30^{\circ}$ consistent with previous results from near-IR interferometry (Millan-Gabet et al. 2001; Eisner et al. 2004), scattered light images (Grady et al. 1999; Fukagawa et al. 2004), and millimeter interferometry (Corder et al. 2005). We therefore adopt a face-on orientation. From the best-fit star+ring model shown in Figure 1, it can be seen (as mentioned above) that the curve does not extrapolate to $V(0)=1.0$, and therefore the model allows for a fraction $f_{i}$ of the total flux to be completely incoherent (i.e., large compared to the angular resolution of the shortest baselines). The best-fit solution [to $V^{2}$ only, resulting in $\chi_{v}^{2}\left(V^{2}\right)=0.9$ ] has the following parameters: $D_{\text {ring }}=3.1 \pm 0.1$ mas $(0.39 \pm$ $0.02 \mathrm{AU}$ ), $f_{i}=0.08 \pm 0.01$ (see dashed lines in Figs. 1 and 2 ). This value for the ring diameter is in good agreement with recent interferometer measurements (Eisner et al. 2004).
The above model describes some of the main features of the $\mathrm{AB}$ Aur system (number of components needed, characteristic size and shape). However, in order to also fit the nonzero CPs, we need to consider nonsymmetric models. As described in $\S 1$, flared disks with nonzero inclinations are expected to occult the near side of the inner disk, resulting in a nonsymmetric brightness. However, as just discussed, the inclination of the $\mathrm{AB}$ Aur disk is believed to be low. Moreover, in order to measure the $\mathrm{CP}$ resulting from asymmetries in the inner disk, the emission has to be well resolved. As shown in Monnier et al. (2006), the $\mathrm{CP}$ is strongly suppressed when, as in the $\mathrm{AB}$ Aur case, the inner disk diameter (3.1 mas) is less than $\frac{1}{2}$ the fringe spacing corresponding to the longest baseline in a triangle. Here we have confirmed this result by introducing an azimuthal cosine modulation of the Gaussian ring in order to approximate the morphology of a partially occulted inner disk (see Monnier et al. 2006 for details of these skewed ring models), and we found that it can produce at most a signal of $\mathrm{CP}(\mathrm{A} 25 \mathrm{~B} 15 \mathrm{C} 10) \sim-2^{\circ}$ when the solutions are constrained to reproduce (even approximately) the $V^{2}$ data and the zero $\mathrm{CP}$ measured in the other baseline triangles.

We therefore must add an additional source component at larger spatial scales that introduces additional skewness to the model. In order to quantify the level of asymmetry that is required, we have adopted a simple Gaussian profile that is offset with respect to the central disk emission ("disk hot spot" model). The new component is thus characterized by its fullwidth at half-maximum (FWHM; $W_{\mathrm{G}}$ ), location (angular separation $r_{\mathrm{G}}$ and position angle P.A. $\left.{ }_{\mathrm{G}}\right)$ and the fractional flux $\left(f_{\mathrm{G}}\right)$ it contributes. Fitting this simple model, we find that solutions exist that reproduce both the $V^{2}$ and $\mathrm{CP}$ data reasonably well. However, we emphasize that several statistically equivalent solutions exist, and although we can assign definite spatial scales and fractional fluxes to the offset Gaussian, we are not able to assign a definite direction to the asymmetry (e.g., there are reasonable solutions with northern as well as southern offsets). We have included the best-fit parameters associated with a few of the best solutions in Table 1 for reference.

Quantitatively, the solutions found are defined by the following characteristics: (1) when the offset Gaussian feature is allowed to be spatially extended, the angular separations are $r_{\mathrm{G}} \simeq 30$ mas $(4.3 \mathrm{AU})$, width $W_{\mathrm{G}} \simeq 12$ mas $(1.7 \mathrm{AU})$, and fractional flux $f_{\mathrm{G}} \simeq 8 \%$ (we also note that in this case, slightly better fits are found when the inner ring is also allowed to be skewed rather than symmetric); and (2) for the special case that the offset component is unresolved (i.e., a point-source companion), closer separations $r_{\mathrm{G}} \simeq 9$ mas $(1.25 \mathrm{AU})$ and lower fractional fluxes $f_{\mathrm{G}} \simeq 2 \%$ are found (in this case with no benefit from additional skew in the inner ring). An example fit 
of the latter case is shown (solid lines) in Figures 1 and 2. The example shown is typical of the quality of the fits obtained $\left[\chi_{\nu}^{2}\left(V^{2}, \mathrm{CP}\right)=1.5\right]$ and corresponds to the parameters $f_{\mathrm{G}}=$ $0.02, r_{\mathrm{G}}=9$ mas $(1.4 \mathrm{AU})$, and P.A. ${ }_{\mathrm{G}}=22^{\circ}$. As can be seen, although the solution is not entirely adequate on all baseline pairs and triangles, indicating the need for a more complex model, this simple model is capable of reproducing most data well, in particular, the observed $\mathrm{CP}(\mathrm{A} 25 \mathrm{~B} 15 \mathrm{C} 10)=-4^{\circ}$.

\section{DISCUSSION}

The principal result of this Letter is the clear detection of an unexpected localized asymmetry in the circumstellar environment of $\mathrm{AB}$ Aur at spatial scales of 1-4 AU; this is an intermediate scale between the inner wall containing the hottest dust and the outer disk.

We first considered that the physical origin of this emission could be $H$-band scattering off the disk atmosphere or "halo." The required asymmetry could easily arise from a small photocenter shift due to, e.g., forward scattering in combination with a small disk inclination. However, this emission would arise from large angular scales (up to $\sim 1^{\prime \prime}$ ) and would be completely resolved by even the shortest IOTA baselines, and therefore could not result in a CP signal. Indeed, the model fitting described above shows that the offset Gaussian must be relatively compact ( $\$ 12$ mas or $1.7 \mathrm{AU}$ ), and it is difficult to reconcile the required amount of flux with scattering over such a small area. Due to the low inclination known for the AB Aur disk, we also do not favor outer disk occultation or opacity effects.

A more likely explanation is that the asymmetry is due to compact thermal emission. The presence and persistence of such a local inhomogeneity in the AB Aur disk at these spatial scales would, however, be surprising, given that the dynamical timescales are very short ( $1-5 \mathrm{yr})$ compared to the age of the system ( 1-4 Myr). A possible explanation is that the disk is not steady state, and the feature corresponds to a gravitational instability, possibly associated with an inner extension of the spiral density waves seen in near-IR coronagraphic and millimeter interferometry images (Fukagawa et al. 2004; Pietu et al. 2005). The existence of a disk instability at few AU radii and for a disk of this age clearly has important implications for the formation of planets in these disks.

Alternatively, we could be detecting a companion embedded in the AB Aur disk. ${ }^{10}$ However, a stellar companion at the few AU separation we infer is unlikely, given that a substantial circumstellar disk has in fact survived around $\mathrm{AB}$ Aur. The intriguing possibility therefore arises that the companion is instead a protoplanetary object forming in the disk.

Similar claims (but based on visibility amplitudes rather than on the more robust $\mathrm{CP}$ ) for a disk thermal instability or close companion have recently been made for the active-disk system FU Orionis (Malbet et al. 2005). We also note the possible connection between the extended structure detected here and the intriguing compact halo (45 mas FWHM) seen in the T Tauri object DG Tau in lunar occultation observations by Leinert et al. (1991). As discussed by Monnier et al. (2006), disk "halos" appear to be common (although not the norm) in young star+disk systems, and they deserve new observational and modeling scrutiny.

Our hypothesis is readily testable. If the nonzero $\mathrm{CP}$ arises in a "disk hot spot," variability in the CP signal over $1-5 \mathrm{yr}$ should reveal the orbital motion that could be followed using short or long baselines from the VLTI AMBER (Malbet et al. 2004) or MIDI (Leinert et al. 2003) instruments, respectively. The angular scales involved also make this object a prime target for direct imaging using high-resolution techniques on large single-aperture telescopes.

The authors gratefully acknowledge support from SAO, NASA (for third telescope development and NNG05G1180G), the NSF (AST-0138303, AST-0352723, for work on imaging with IOTA), and the Jet Propulsion Laboratory (JPL awards 1236050 and 1248252). The IONIC-3 instrument has been developed by LAOG and LETI in the context of the IONIC collaboration (LAOG, IMEP, LETI). The IONIC project is funded by the CNRS (France) and CNES (France).

\footnotetext{
${ }^{10}$ We note that Baines et al. (2006) report "spectroastrometric" evidence of binarity in AB Aur (and several other HAeBe objects). However, they infer large separations of $0.5-3$." 0 ; therefore this putative companion cannot be related to the $\mathrm{CP}$ signatures modeled here.
}

\section{REFERENCES}

Akeson, R. L., Ciardi, D. R., van Belle, G. T., \& Creech-Eakman, M. J. 2002, ApJ, 566, 1124

Akeson, R. L., Ciardi, D. R., van Belle, G. T., Creech-Eakman, M. J., \& Lada, E. A. 2000, ApJ, 543, 313

Akeson, R. L., et al. 2005, ApJ, 622, 440

Baines, D., Oudmaijer, R. D., Porter, J. M., \& Pozzo, M. 2006, MNRAS, 367, 737

Baldwin, J. E., et al. 1996, A\&A, 306, L13

Berger, J-P., et al. 2003, Proc. SPIE, 4838, 1099

Corder, S., Eisner, J., \& Sargent, A. 2005, ApJ, 622, L133

Cornwell, T. J., \& Wilkinson, P. N. 1981, MNRAS, 196, 1067

Coudé du Foresto, V., Ridgway, S., \& Mariotti, J. M. 1997, A\&AS, 121, 379

Dullemond, C. P., Dominik, C., \& Natta, A. 2001, ApJ, 560, 957

Eisner, J. A., Hillenbrand, L. A., White, R. J., Akeson, R. L., \& Sargent, A. I. 2005, ApJ, 623, 952

Eisner, J. A., Lane, B. F., Hillenbrand, L. A., Akeson, R. L., \& Sargent, A. I. 2004, ApJ, 613, 1049

Fukagawa, M., et al. 2004, ApJ, 605, L53

Grady, C. A., Woodgate, B., Bruhweiler, F. C., Boggess, A., Plait, P., Lindler, D. J., Clampin, M., \& Kalas, P. 1999, ApJ, 523, L151

Isella, A., \& Natta, A. 2005, A\&A, 438, 899

Jennison, R. C. 1958, MNRAS, 118, 276

Kenyon, S. J., \& Hartmann, L. 1987, ApJ, 323, 714

Kraus, S., et al. 2005, AJ, 130, 246

Leinert, Ch., Haas, M., Mundt, R., Richichi, A., \& Zinnecker, H. 1991, A\&A, 250,407
Leinert, Ch., et al. 2003, Ap\&SS, 286, 73

Malbet, F., Lachaume, R., \& Monin, J. L. 2001, A\&A, 379, 515

Malbet F., et al. 2004, Proc. SPIE, 5491, 1722 2005, A\&A, 437, 627

Millan-Gabet, R., Malbet, F., Akeson, R., Leinert, Ch., Monnier, J., \& Waters, R. 2006, in Protostars and Planets V, ed. B. Reipurth, D. Jewitt, \& K. Keil (Tucson: Univ. Arizona Press), in press (astro-ph/0603554)

Millan-Gabet, R., Schloerb, F. P., \& Traub, W. A. 2001, ApJ, 546, 358

Millan-Gabet, R., Schloerb, F. P., Traub, W. A., Malbet, F., Berger, J. P., \&

Bregman, J. D. 1999, ApJ, 513, L131

Monnier, J. D. 2003, Rep. Prog. Phys., 66, 789

Monnier, J. D., \& Millan-Gabet, R. 2002, ApJ, 579, 694

Monnier, J. D., et al. 2004, ApJ, 602, L57

$$
\text { 2005, ApJ, 624, } 832 \text { (erratum 632, 689) }
$$$$
\text { 2006, ApJ, in press }
$$

Muzerolle, J., Calvet, N., Hartmann, L., \& D'Alessio, P. 2003, ApJ, 597, L149

Natta, A., Prusti, T., Neri, R., Wooden, D., Grinin, V. P., \& Mannings, V. 2001, A\&A, 371, 186

Pauls, T., Young, J. S., Cotton, W. D., \& Monnier, J. D. 2005, PASP, 117, 1255

Pedretti, E., et al. 2005, Appl. Opt., 44, 5173

Pietu, V., Guilloteau, S., \& Dutrey, A. 2005, A\&A, 443, 945

Readhead, A. C. S., Walker, R. C., Pearson, T. J., \& Cohen, M. H. 1980, Nature, 285, 137

Traub, W. A., et al. 2003, Proc. SPIE, 4838, 45

Tuthill, P. G., Monnier, J. D., \& Danchi, W. C. 2001, Nature, 409, 1012 